\title{
STRUCTURE AND KINEMATICS IN THE NORTHEASTERN OUTER PARTS OF THE SMC
}

\author{
D. HATZIDIMITRIOU, R.D. CANNON \\ Anglo-Australian Observatory \\ Epping NSW 2121 \\ Australia \\ M.R.S. HAWKINS \\ Royal Observatory Edinburgh \\ Blackford Hill \\ Edinburgh EH9 $3 \mathrm{HJ}$ \\ Scotland
}

\begin{abstract}
We present preliminary results from a study of the radial velocities of red horizontal branch stars in the north-eastern outer parts of the Small Magellanic Cloud (SMC). The sample is located in an area with a large line-of-sight depth of $\simeq 15 \mathrm{kpc}$. It is suggested that there is a correlation between line-ofsight distance and radial velocity for these stars, with the more distant ones showing significantly higher velocities.
\end{abstract}

\section{Introduction}

In a recent paper (Hatzidimitriou and Hawkins 1989 - HH), we studied the line-of-sight depth as a function of position on the sky for the intermediate and old populations ( $\tau \geq 1 \mathrm{Gyr}$ ) of the SMC, over an area of 48 square degrees in the NE and W-SW outer parts (projected radial distance from the centre of the $S M C>2 \mathrm{kpc}$ ). The study consisted of the construction of colour magnitude diagrams over the entire area. The red horizontal branch (clump) stars were used as distance indicators (which is a valid method, since there are no populations younger than $5 \times 10^{8} \mathrm{yr}$ in these areas). It was found that while in the W-SW the SMC seems to be confined well within its tidal radius, and with a uniform distance modulus, in the $\mathrm{NE}$ (well to the North of the Wing) there is a much larger depth along the line-of-sight reaching values of $15-20 \mathrm{kpc}$ in the North of the field. At the same time the mean distance modulus decreases by $2.5 \mathrm{kpc} / \mathrm{deg}$ as we move away from the centre. The effect was interpreted as resulting from the tidal disruption of the SMC by the LMC. The aim of the present work was the study of the kinematics of these stars in the area of maximum depth.

\section{Observations}

The sample of the stars observed consisted of 50 horizontal branch/clump stars, selected from the colour-magnitude diagrams of $\mathrm{HH}$. The stars were distributed over a 40 arcmin field, located outside the SMC main mass distribution $(\mathrm{RA}=12100, \mathrm{dec}=-704800(1950)$ ) at $\sim 3 \mathrm{kpc}$ from 
the optical centre of the SMC to the North-East. The area has a mean distance modulus of $(\mathrm{m}-\mathrm{M})_{0}$ $=18.70$, and an estimated line-of-sight depth of $\sim 15 \mathrm{kpc}(\mathrm{HH})$.

The observations took place in December 1989, at the Anglo-Australian Telescope, using the multiobject spectroscopic facility AutoFib with the RGO spectrograph, and the IPCS as a detector. The wavelength region studied was between $3700-4700 \AA$ and the resolution was 70 $\mathrm{km} / \mathrm{sec}$. The data were reduced using standard routines in the FIGARO software package. At the completion of the reduction process only 15 of the stars in the sample had sufficient signal-to-noise ratio (due to adverse observing conditions) to justify further analysis. To determine relative radial velocities, the spectra of the SMC stars were cross-correlated with the high signal-to-noise spectra of five radial velocity standards. The zero-point of the velocity system was found to be accurate to within $5 \mathrm{~km} / \mathrm{sec}$. The velocities derived for the 15 stars in our sample have accuracies $\sim 10-15$ $\mathrm{km} / \mathrm{sec}$.

\section{Results}

The velocities derived with the previously mentioned method for the stars in our sample, range from $120 \mathrm{~km} / \mathrm{sec}$ to $260 \mathrm{~km} / \mathrm{sec}$ (in the local frame of reference), with a mean of $160 \mathrm{~km} / \mathrm{sec}$, with the exception of one star which has a velocity of $80 \mathrm{~km} / \mathrm{sec}$ and is very likely a foreground star.

Interestingly, there is evidence that the more distant of the observed stars (distances estimated from apparent magnitudes) also have higher velocities. There is good general agreement with the correlation between radial velocity and distance found for Cepheids by Mathewson, Ford and Visvanathan (1988). This correlation suggests an increase in radial velocity by $\sim 4 \mathrm{~km} / \mathrm{sec} / \mathrm{kpc}$, which gives a time scale of expansion of about $3 \times 10^{8} \mathrm{yr}$, in agreement with the collision model of Fujimoto and Murai (1984). The sample is too small for conclusive results, but it seems plausible that these predominantly intermediate/old stars show signs of the same disruptive effects of the LMC on the SMC observed among younger populations.

\section{References}

Fujimoto, M. and Murai, T. (1984), IAU Symp. 108 Structure and Evolution of the Magellanic Clouds, S. van den Bergh and K.S. de Boer (eds). (Reidel: Dordrecht) p.115.

Hatzidimitriou, D. and Hawkins, M.R.S. (1989), Mon. Not. R. Astron. Soc. 241, 667. Mathewson, D.S., Ford, V.L. and Visvanathan, N. (1988), Astrophys. J. 333, 617. 